\title{
Comparison of single plane and biplane radionuclide ventriculograms performed in oblique projections in patients with acute myocardial infarction
}

\author{
DUNCAN DYMOND, DAVID STONE, A. T. ELLIOTT, K. E. BRITTON, \\ S. O. BANIM, AND R. A. J. SPURRELL \\ From the Departments of Cardiology and Nuclear Medicine, St. Bartholomew's Hospital, London
}

SUMMARY Thirty-five patients were studied by first pass radionuclide ventriculography in both right anterior oblique and left anterior oblique projections, after an acute myocardial infarction. Left ventricular ejection fraction was measured in both obliques from the background corrected time-activity curves and the values correlated closely. Mean left ventricular ejection fraction from the two obliques correlated well with that measured from the ventricular perimeters using a biplane area-length formula. End-diastolic volume from the single plane right anterior oblique correlated with that measured using the biplane formula. Combined right and left anterior oblique projections allowed identification of akinetic sites in all patients, whereas the right anterior oblique alone failed to delineate akinetic sites in $1 / 10(10 \%)$ of patients with anterior infarction, $2 / 9(22 \%)$ with inferior infarction, and 6/16 (37\%) with multiple areas of infarction. The left anterior oblique alone identified akinesis in all patients with anterior infarction, but failed in $2 / 9(22 \%)$ with inferior infarction and $5 / 16(31 \%)$ with multiple sites of infarction. The mean percentage akinesis from both obliques showed a significant negative correlation with mean ejection fraction as did that from the right anterior oblique alone. Percentage akinesis from the left anterior oblique alone correlated less well with left ventricular ejection fraction. The combination of right and left anterior oblique radionuclide ventriculograms provides greater accuracy in the detection of akinetic segments, especially when multiple areas of infarction are present. The use of a single plane left anterior oblique alone may prove misleading in the quantification of wall motion abnormalities, and in this respect should be interpreted with caution.

Since the animal experiments of Tennant and Wiggers (1935), abnormal regional myocardial performance has been thought to be a cause of ventricular dysfunction. In 1967 Herman et al. showed conclusively that localised abnormalities of ventricular contraction could exist in man and suggested them as a cause of clinical cardiac failure. Feild et al. (1972) described a quantitative relation between the degree of myocardial damage, as assessed by biplane contrast cineangiography, and the presence of cardiac failure. The development of radionuclide techniques capable of providing information on both ejection fraction and regional wall motion has been a major advance, allowing the non-invasive acquisition of such data, and previous reports have indicated the applicability of radio-

Received for publication 25 April 1979 nuclide angiograms to the study of patients with myocardial infarction (Kostuk et al., 1973; Rigo et al., 1974; Schelbert et al., 1976). In patients with ischaemic heart disease the right and left anterior oblique have been regarded as the optimum projections for assessing wall motion abnormalities (Cohn et al., 1974), but isotope techniques that employ scanning of the radioactive cardiac blood pool with the aid of electrocardiographic gating devices are limited in the choice of projection, because of inadequate spatial separation of the ventricles which contain tracer simultaneously at equilibrium. In these cases, the left anterior oblique has been the projection of choice for wall motion analysis, though even then visualisation of the inferior and anterior walls of the left ventricle may be suboptimal when isotope is present simultaneously in both ventricles (Pierson et al., 1977). 
The first pass radionuclide ventriculogram provides temporal separation of the cardiac chambers as counts are accumulated only during the initial passage of the tracer through the central circulation. The problem of overlap in the right anterior oblique is thus not present, and choice of projection is not a limiting factor.

On the basis of this, a study was undertaken to examine the ability of first pass radionuclide ventriculograms performed in both right and left projections to provide information on global and segmental ventricular function in patients with acute myocardial infarction.

\section{Patients and methods}

Thirty-five patients were studied between five and eight days after an acute myocardial infarction. There were 33 men and two women, with a mean age of 56, and an age range of 33 to 73 years. Myocardial infarction was diagnosed by the conventional criteria of typical history, evolution of electrocardiographic $Q$ waves, and characteristic rise and fall of serum enzymes. The site of infarction was read from the patients' electrocardiograms by an independent observer, and studies were analysed either by a cardiologist or a trained technician unaware of the site of infarction. No patient was studied while still requiring acute coronary care.

Radionuclide ventriculography was carried out using a computerised multi-crystal gamma camera (Baird-Atomic, system 77) with high count rate capabilities (Budinger and Rollo, 1977). Studies were performed using a $1 \cdot 5$-inch parallel hole collimator. A bolus of high specific activity ${ }^{99 \mathrm{~m}}$ Technetium pertechnetate was injected into a medial vein of the right arm, followed by a rapid $20 \mathrm{ml}$ saline flush, as previously described (Dymond et al., 1979). Data were collected in frame mode at 0.05 -second intervals for 50 seconds, stored on magnetic disc, and transferred from disc to magnetic tape for permanent storage. The first study was performed in the $30^{\circ}$ right anterior oblique projection, and 10 to 15 minutes allowed to elapse so that adequate blood pool clearance of the first bolus could occur. The patient was then positioned in the $30^{\circ}$ left anterior oblique projection with a $15^{\circ}$ caudal tilt. Before the second bolus injection a static background frame was collected to a maximum of 9000 counts per cell. Immediately after this frame was obtained the second bolus of ${ }^{99 \mathrm{~m}} \mathrm{~T}$ echnetium was injected, and counting again carried out for 50 seconds. A $10 \mathrm{mCi}$ bolus was used for the first injection and $15 \mathrm{mCi}$ for the second. The first study was corrected for the dead-time of the instrument using a manufacturer supplied computer programme, and for the field non-uniformity by automatically correcting each frame of data for differences between individual crystals, using a uniform flood source. The second study was corrected for residual blood pool activity as well as for dead-time and non-uniformity.

Analysis of each study was carried out by first replaying the study in the form of 12 serial images, each representing 30 summed 0.05 .second frames and the left ventricular phase identified. A region of interest was drawn using a magnetic pen and grid array, the latter representing the detector's 294 crystals. A 0.05 second per frame time-activity curve was generated. Left ventricular ejection fraction was calculated for both obliques from the change in counts from end-diastole to end-systole, the counts in the ventricle being proportional to volumes at any one time. The formula for ejection fraction takes into account the contribution of background non-ventricular counts, background frames being chosen directly from the flat part of the left ventricular time-activity curve approximately 0.5 seconds before the peaks and troughs of the laevophase (Dymond et al., 1979). This method for calculating ejection fraction makes no assumptions about ventricular shape. The peaks and troughs were then summed by the computer to provide one representative cardiac cycle, with high count rates for statistical accuracy. Ventricular perimeters for each oblique were generated by computer, using an isocount edge detection technique whereby the 5 per cent count band corresponding to 25 or 30 per cent of the maximum end-diastolic count per cell is enhanced. Perimeters for end-diastole and end-systole were superimposed. For the calculation of ventricular volumes, the formula of Greene et al. (1967) was applied to the right anterior oblique perimeters. For the combined right and left anterior oblique studies, volumes were calculated according to the method of Wynne et al. (1978), using the areas (A) of the cardiac chambers in both right and left obliques, and the long axes (L), the longest measured chord in any direction within the ventricular silhouette.

Thus volume in millilitres from both obliques

$$
=8 / 3 \pi \times \frac{\text { Area }(\mathrm{RAO}) \times \text { Area }(\mathrm{LAO})}{\mathrm{L}_{\min }}
$$

where $\mathrm{L}_{\min }$ is the smaller of the two long axes from either left or right oblique. All areas and lengths were measured by tracing on to a pressure-sensitive pad linked to a computer and an algorithm analogous to planimetry, as previously described 
(Dymond et al., 1979). In addition the extent of akinesis produced by the infarction was measured by expressing the length for which the end-diastolic and end-systolic perimeters were exactly superimposed, indicating no inward wall motion, as a percentage of the total end-diastolic circumference (Feild et al., 1972).

\section{Results}

Ten patients had suffered anterior infarction, nine inferior, and 16 patients were judged to have multiple areas of infarction. Ejection fraction calculated from the time-activity curve of the right anterior oblique study ranged from 20 to 71 per cent, with a mean of 39.4 per cent. The values from the left anterior oblique projection ranged from 20 to 60 per cent, with a mean of $34 \cdot 2$ per cent. Fig. 1

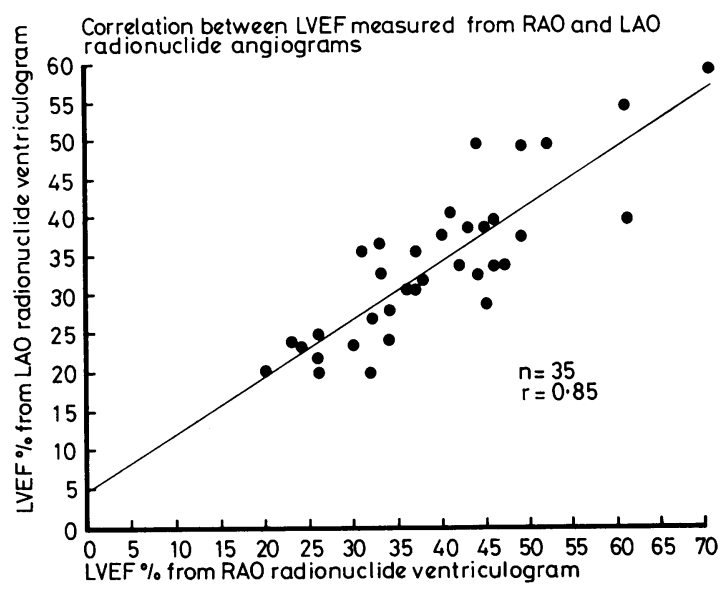

Fig. 1 Correlation between ejection fractions (LVEF) measured independently from time-activity curves for right anterior oblique (RAO) and left anterior oblique (LAO) studies.

shows the relation between these two independently obtained values. There is a good correlation with a coefficient of 0.85 which is highly significant $(P<0.001)$. Analysis of the regression line by Student's $t$ test indicates that the slope is significantly different from one and that the values of ejection fraction obtained from the left anterior oblique (LAO) are significantly lower than that from the right anterior oblique (RAO), with a regression equation:

$$
\text { LVEF }(\text { LAO })=0.757 \text { LVEF }(\text { RAO })+4.378 .
$$

The intercept of the graph is not significantly different from zero.
Fig. 2 shows the correlation between the mean ejection fraction from the two obliques and the ejection fraction obtained from the biplane geometric formula. In the 27 patients in whom adequate

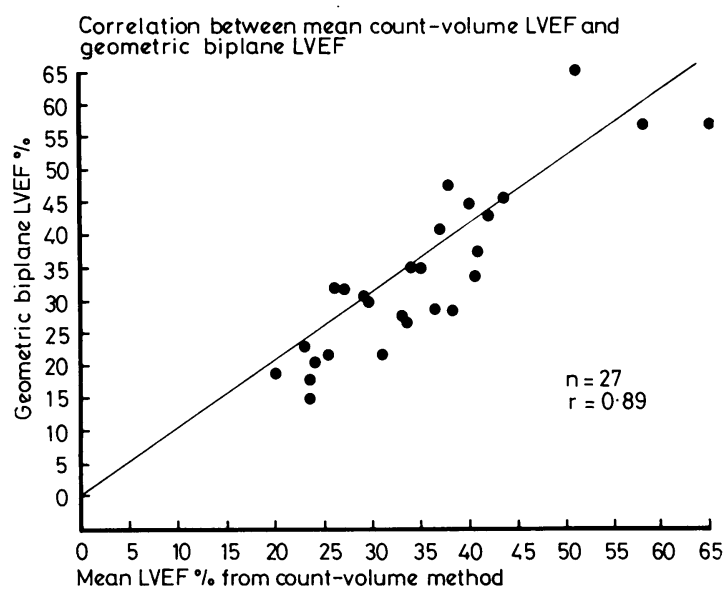

Fig. 2 Correlation between mean ejection fraction ( $L V E F$ ) obtained from the left anterior oblique (LAO) and right anterior oblique ( $R A O)$ study, with that measured from ventricular perimeters of both obliques.

measurements could be made, the correlation is significant, with a coefficient of $0.89(\mathrm{P}<0.001)$. Analysis of this graph by Student's $t$ test showed that the slope did not vary significantly from one and the intercept did not vary significantly from zero.

End-diastolic volume measured from the biplane formula correlated closely with the volume from the single plane right anterior oblique $(r=0.88$, $P<0.001$ ), as shown in Fig. 3. Despite the

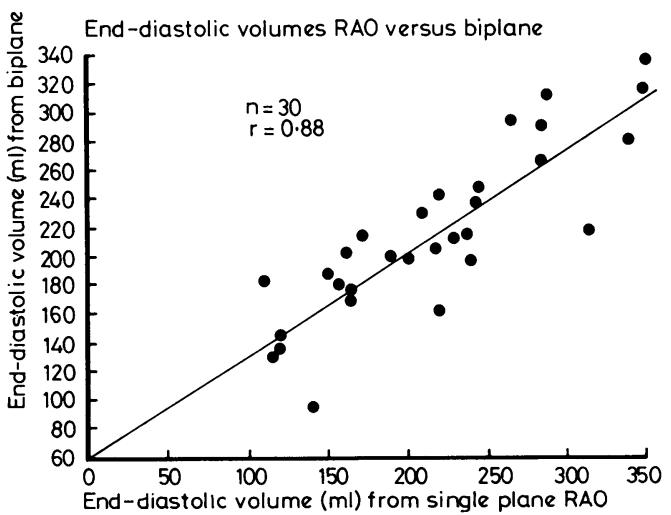

Fig. 3 Correlation between end-diastolic volume measured from the single plane right anterior oblique with that measured from both obliques. 
significant regression coefficient, there was distinct disparity between the absolute values for volumes, with a regression equation of $y=0 \cdot 716 x+62 \cdot 6$.

For the purpose of wall motion analysis, five ventricular segments were considered as described by Cohn et al. (1974). The right anterior oblique was used to identify three segments, namely anterior, apical, and inferior. The left anterior oblique was able to provide information on two segments, septal and posterolateral. The comparative ability of either oblique to detect akinesis is shown in Table 1.

Table 1 Comparative ability of single plane and biplane projections to detect akinesis in anterior, inferior, and multiple infarctions

\begin{tabular}{lcccc}
\hline Infarct & Number & $R A O$ & LAO & Biplane \\
\hline Anterior & 10 & 9 & 10 & 10 \\
Inferior & 9 & 7 & 7 & 9 \\
Combined & 16 & 10 & 11 & 16 \\
\hline
\end{tabular}

RAO, right anterior oblique; LAO, left anterior oblique.

Of 10 patients with anterior infarction, the right anterior oblique projection detected akinesis anteriorly in nine $(90 \%)$, whereas the left anterior oblique projection successfully identified akinesis in all 10. The right anterior oblique alone and the left anterior oblique alone each demonstrated akinesis in seven of the nine $(78 \%)$ with inferior infarction, the left anterior oblique showing akinesis in the lower part of the posterior wall and apex. Combination of the two obliques allowed identification of akinetic sites in all nine.

In 16 patients with multiple sites of infarction, the right anterior oblique demonstrated akinesis in more than one segment in $10(62 \%)$, the left anterior oblique in $11(68 \%)$, and the combined projections were successful in all 16 cases.

Fig. 4 is an example of right anterior oblique and left anterior oblique radionuclide ventriculograms in a patient with anterior and posteroinferior infarction electrocardiographically. The left-hand image is the superimposed perimeters of the right anterior oblique study, the right-hand image the left anterior oblique. The end-diastolic perimeter is shown in each case in blue, the end-systolic perimeter in yellow. The right anterior oblique study shows an area of akinesis anteriorly, while the inferior region shows definite inward motion in systole. The left anterior oblique shows the posterolateral wall to be totally akinetic, whereas the septum shows motion in systole. Thus each projection identified an area of akinesis exclusively, and the complete information on segmental wall motion would not have been obtained from a single projection alone.

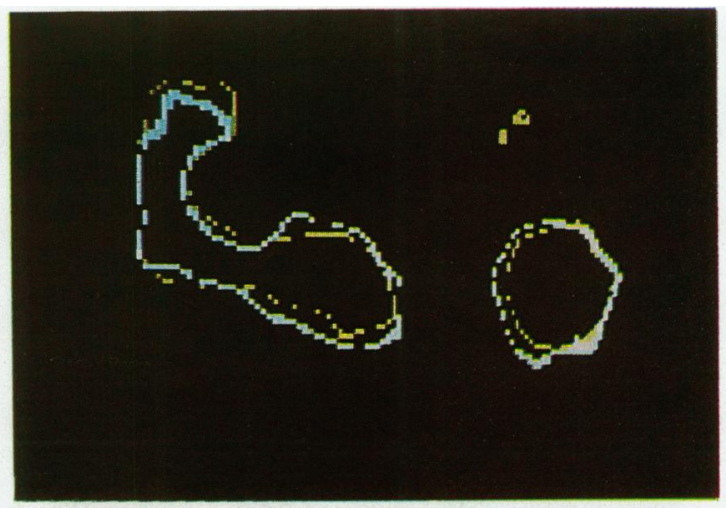

Fig. 4 Perimeters of the right anterior oblique (RAO) (left-hand image) and left anterior oblique ( $L A O)$ radionuclide ventriculograms from a patient with anterior and posteroinferior infarction. End-diastolic perimeter is in blue; end-systolic perimeter in yellow. RAO only shows akinesis anteriorly, the LAO only posterolaterally.

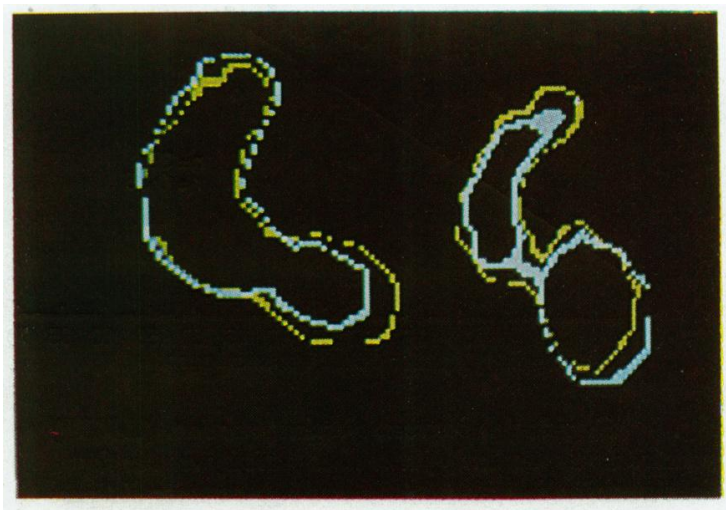

Fig. 5 Perimeters of the right anterior oblique (RAO) (left-hand image) and left anterior oblique (LAO) radionuclide ventriculograms from a patient with localised anteroseptal infarction. No akinesis on RAO, but small area of akinesis shown septally on $L A O$.

Fig. 5 is an example of a right and left anterior oblique study in a patient with localised anteroseptal infarction electrocardiographically. The left-hand image, the right anterior oblique, shows no akinesis, whereas the left anterior oblique study shows a small area of akinesis septally.

The relation between the extent of akinesis from each study and the degree of depression of overall ventricular function as measured by ejection fraction, is shown in Fig. 6a, 6b, and 6c. In Fig. 6a the 
a Correlation between mean akinetic length and mean LVEF

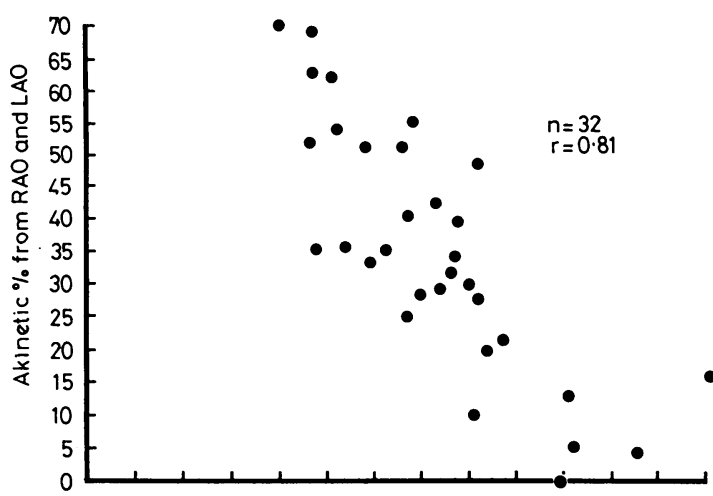

b Correlation between akinetic \% from single plane RAO and mean LVEF
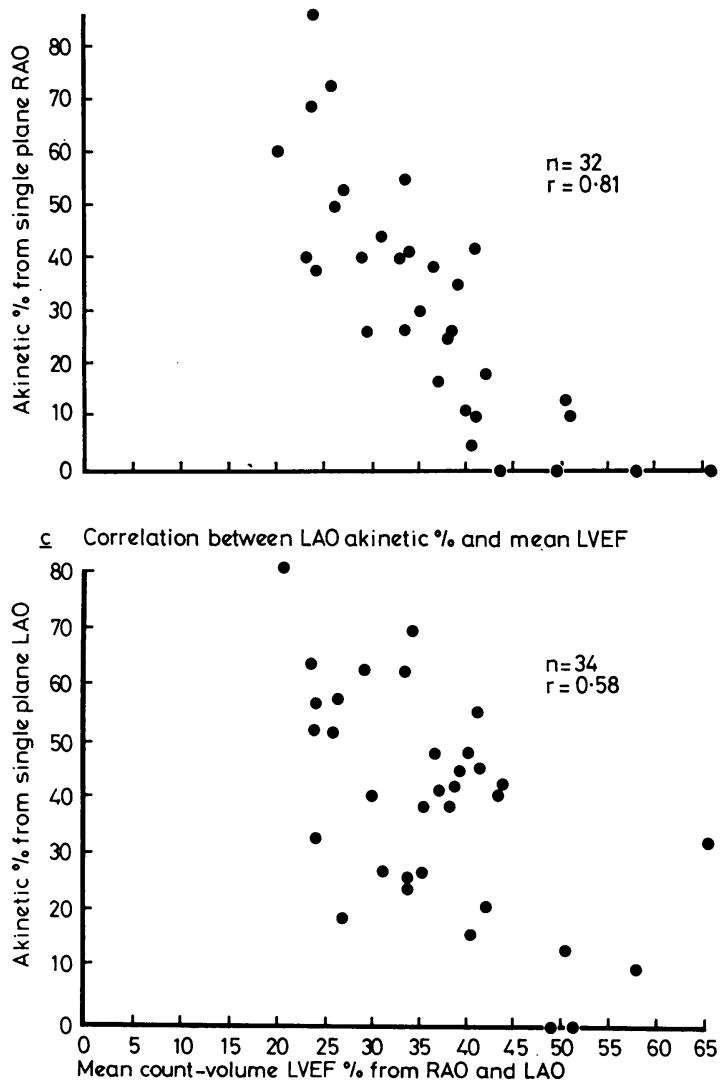

Fig. 6 Relation of mean ejection fraction from two obliques (LVEF) to (a) akinetic percentage (mean derived from $R A O$ and $L A O$; (b) akinetic percentage from single plane $R A O$; and (c) akinetic percentage from single plane $L A O$.

mean ejection fraction and akinetic percentage from the two obliques are plotted for 32 patients. There is a significant negative correlation with a coefficient of $0.81 \quad(P<0.001)$. Mean ejection fraction and akinesis from a single plane right anterior oblique are plotted in Fig. 6b. Again, there is a significant negative correlation with an identical coefficient as for the biplane study $(r=0.81, P<0.001)$. Analysis of the relation between the two indices for the single plane left anterior oblique yielded a much less good correlation, with a coefficient of $0.58(\mathrm{P}<0.005)$ and a large amount of scatter (Fig. 6c).

For the entire patient population percentage akinesis from the right anterior oblique was 31.9 \pm 22.6 per cent (mean \pm standard deviation), and for the left anterior oblique the value was 39.6 \pm 20.5 per cent. This difference is statistically significant $(P<0.05)$ suggesting that the left anterior oblique overestimates the extent of akinesis compared with the right anterior oblique. Table 2 shows the mean percentage akinesis with standard deviations, in patients with different sites of infarction. In none of the three subgroups, inferior, anterior, or multiple infarction, did the differences in mean akinetic percentage reach statistical significance despite the large discrepancies between the means from the two obliques in patients with inferior and multiple infarctions.

Table 2 Comparison of percentage akinesis from two obliques for whole group and for anterior, inferior, and multiple infarctions

\begin{tabular}{lll}
\hline & $\begin{array}{l}R A O \\
\text { (mean } \pm S D)\end{array}$ & $L A O($ mean $\pm S D)$ \\
\hline Inferior infarct & $15.4 \pm 14.6 \%$ & $28.4 \pm 25.5 \%$ (NS) \\
Anterior infarct & $33.1 \pm 17.8 \%$ & $32.2 \pm 14.9 \%$ (NS) \\
Multiple infarcts & $38.5 \pm 25 \cdot 1 \%$ & $49.8 \pm 14.9 \%(\mathrm{NS})$ \\
Whole group & $31.9 \pm 22.6 \%$ & $39.6 \pm 20.5 \%(\mathrm{P}<0.05)$
\end{tabular}

RAO, right anterior oblique; LAO, left anterior oblique; SD, standard deviation.

Percentage akinesis was greatest in those with multiple infarctions whether from the right anterior oblique or left anterior oblique, and similarly both projections showed a greater percentage akinesis in patients with anterior infarction than in those with inferior infarction. Analysis of these differences by a non-paired $t$ test showed that for both the right and the left anterior oblique, akinesis in anterior infarction failed to be significantly greater than that in inferior infarction. For the right anterior oblique, percentage akinesis was not significantly higher in multiple than in anterior infarction, though the difference between multiple and inferior infarction did achieve significance $(P<0.02)$. For the left anterior oblique, however, percentage akinesis was significantly greater for multiple than for anterior infarction $(P<0.02)$, and for inferior infarction $(P<0.02)$. 


\section{Discussion}

Several previous studies have pointed out the value of radionuclide techniques in the evaluation of patients with myocardial infarction. The right anterior oblique projection has been utilised for measurements of ejection fraction from timeactivity curves (Steele et al., 1974; Henning et al., 1975; Schelbert et al., 1976), but the two latter studies employed radarkymography for wall motion analysis. Kostuk et al. (1973) described the detection of wall motion abnormalities in the left lateral projection using peripheral venous injection of tracer. However, it is well recognised that in patients with ischaemic heart disease the right anterior oblique and left anterior oblique projections are the optimal for studying regional abnormalities of wall motion (Cohn et al., 1974; Miller et al., 1974). Gated blood pool scanning has been performed in right and left anterior oblique projections in patients with infarction (Rigo et al., 1974) and ejection fraction and wall motion quantified, though no comparison was made between the right and left anterior oblique in that study. Because of the anatomical overlap of right and left ventricles in the right anterior oblique, changes in radioactive counts over the left ventricular region of interest will contain some data from the right ventricular blood pool when gated equilibrium imaging is employed in the right anterior oblique (Pierson et al., 1977). The first pass radionuclide ventriculogram, by providing temporal separation of the ventricles, theoretically eliminates the contribution of right ventricular counts to the left ventricular timeactivity curve. Previous reports have described contrast angiographic correlations of right anterior oblique radionuclide studies using the multicrystal gamma camera, which has the ability to accumulate enough counts during the first pass to provide statistically accurate data on wall motion (Bodenheimer et al., 1978; Dymond et al., 1979). It was not the purpose of this study to relate the angiograms of individual patients to clinical status, as it is already well recognised that the extent of left ventricular damage is related to functional capacity (Feild et al., 1972). Instead, an attempt has been made to compare the right and left anterior obliques and to examine the ability of the combined projections to provide clinically relevant information.

The correlation obtained between ejection fraction measured independently from the two obliques is similar to that found by Bodenheimer et al. (1978), though these workers employed a 35 per cent background subtraction to the right anterior oblique time-activity curve on the basis that this provided the best correlation with left anterior oblique ejection fraction. In this present study no such standard background subtraction was used, rather the subtraction was carried out on each individual time-activity curve as described above. Marshall et al. (1977) found that ejection fraction from the anterior view was significantly higher than that from the left anterior oblique, using a similar background correction as was used in this study. The difference, as in this study, may well be related to variations in the efficiency of detecting background radioactivity from the lungs and the left atrium in the two positions. It is also possible that in the right anterior oblique some lag of activity in the right ventricle may contribute to the background portion of the time-activity curve and thus the background may be overestimated leading to an inappropriately high ejection fraction. In the left anterior oblique the spatial separation between the ventricles would preclude this. The main assumption in this method of background correction is that the background remains relatively constant during the left ventricular phase, compared with the gross fluctuation in counts between diastole and systole. This assumption is supported by the results of Van Dyke et al. (1972). The theoretical objections to the assumption appear to be of little importance practically in view of the previous good correlations obtained with ejection fraction measured by contrast angiography. Indeed, though it might be logical to expect the left anterior oblique to provide the closer correlation with contrast angiography in view of the problems with background, this is not always the case (Garcia et al., 1977).

Calculation of ejection fraction by area-length formulae depends on the assumption that the ventricle conforms to certain geometric shapes, whereas ejection fraction measured from changes in radioactive counts does not. Mean ejection fraction from the count-volume method correlated well with that calculated from the biplane geometric formula applied to the ventricular perimeters. Recently, volumes calculated from biplane oblique ventriculography have been shown to correlate well with true volumes measured from human ventricular casts (Wynne et al., 1978). In the present study the correlation between count-volume ejection fraction and biplane geometric ejection fraction was close, varying by 10 per cent or more in only three patients. This suggests that a biplane formula can be applied to radionuclide ventriculograms as well as contrast angiograms. It is possible that a closer correlation might have been obtained by varying the obliquity of the left anterior oblique and the degree of forward tilt, manoeuvres which alter the long axis of the left anterior oblique and hence calculated volume (Wynne et al., 1978). Though no 
patient in our series underwent contrast angiography, a previous study has shown a close correlation between a geometric ejection fraction from the right and left anterior oblique gated blood pool scans and contrast angiographic ejection fraction (Garcia et al., 1977).

Measurement of absolute left ventricular volume has become a standard technique in the assessment of left ventricular function (Rackley, 1976), and volumes calculated from radionuclide ventriculograms have been shown to correlate with volumes measured from contrast angiograms (Strauss et al., 1971; Kostuk et al., 1973), though in the right anterior oblique radionuclide volumes may overestimate the values compared with contrast angiograms (Dymond et al., 1979). Results in this study suggest that a single plane right anterior oblique will overestimate end-diastolic volumes compared with biplane values when large volumes are present. This finding is in agreement with the contrast data of Cohn et al. (1974) in which the most disparity occurred in patients with asynergy, such as the group described here, despite significant correlation coefficients. Indeed, the regression equation in Cohn's series for patients with coronary artery disease and ventricular asynergy was almost identical to the equation from our own data. Wynne et al. (1978) also found that the biplane study led to less of an overestimation of true volume than a single plane right anterior oblique projection. The accurate assessment of end-diastolic volume is of clinical relevance given the finding of Rigo and coworkers (1974) that end-diastolic volume was only increased in infarct patients when left ventricular filling pressure was increased and cardiac index reduced, findings which relate to prognosis (Mathey et al., 1974).

For the detection and localisation of infarction, akinetic segments from the radionuclide ventriculograms were correlated with the patients' electrocardiograms. The latter have been previously shown to be reliable in defining site of infarction demonstrated angiographically (Miller et al., 1974). In the patients with anterior infarction the right anterior oblique missed akinesis on the one patient with localised anteroseptal infarction (Fig. 5), whereas septal akinesis was detected in all 10 on the left anterior oblique projection. In inferior infarction the left and right anterior oblique projections each detected seven of nine akinetic segments. The two with no akinesis on the right anterior oblique projection had electrocardiographic changes of posteroinferior infarction, and had posterior akinesis on the left anterior oblique projection. Posterior changes would not be detected on the right anterior oblique projection. Of the seven with no posterior changes electrocardiographically, all had inferior akinesis on the right anterior oblique and two had no akinesis on the left anterior oblique. Where posterior infarction occurs, therefore, the left anterior oblique may prove superior to the right anterior oblique, whereas the converse may be true if inferior wall abnormalities predominate. In fact Cohn et al. (1974) found normal left anterior oblique ventriculograms in all five patients with inferior infarction who had asynergy in the right anterior oblique projection. It is possible that the addition of hypokinesis or dyskinesis as criteria for abnormal wall motion might alter the detection rate for the two projections (Miller et al., 1974), and recent reports have indicated that hypokinesis as well as akinesis can be shown from radionuclide ventriculograms (Bodenheimer et al., 1978). This hypokinesis has been well correlated with asynergy from contrast ventriculograms in the right anterior oblique but not the left anterior oblique. In patients with combined areas of infarction, biplane views identified multiple akinetic sites in every case whereas each single plane had a substantial failure rate to detect more than one infarcted segment. Thus, biplane radionuclide ventriculography is more likely to be beneficial in patients with the greatest amount of asynergy, a finding in agreement with that of Cohn et al. (1974).

The extent of akinesis from radionuclide ventriculograms has been previously shown to correlate closely with that from contrast angiograms (Rigo et al., 1974; Dymond et al., 1979). For both right and left anterior oblique projections multiple infarctions produced greater akinesis than anterior infarctions, which in turn had more akinesis than inferior infarctions. These findings are in agreement with those from contrast angiographic data (Feild et al., 1972; Miller et al., 1974) and suggest that more myocardial damage occurs after anterior infarction than after inferior infarction (Sobel et al., 1972). That the differences between the extent of akinesis for anterior and inferior infarctions failed to reach statistical significance in this study is partly related to the small numbers in the groups and the large variability in segment size. Our results in this report are similar to those of Feild et al. (1972) though in this latter study biplane ventriculography was carried out in the anteroposterior and lateral projections which are not the best projections for assessing asynergy.

A quantitative relation between the size of an akinetic segment and ejection fraction has been described in the experimental animal (Pairolero et $a l ., 1970$ ) and in man (Feild et al., 1972; Rigo et al., 1974) but no study has compared results from different projections. 
The advent of the non-invasive radionuclide ventriculogram has produced the potential for simple, safe acquisition of data on ventricular function in a wide variety of diseases. Limitations in choice of projection for technical reasons mean that the advantages and limitations of each projection must be precisely defined to avoid misinterpretation of data. It is evident from the graphs in Fig. 6 that the relation between ejection fraction and akinetic percentage is superior for the biplane or single plane right anterior oblique than for left anterior oblique. The significant difference between extent of akinesis for the left anterior oblique and right anterior oblique suggests that the left anterior oblique will tend to overestimate the extent of myocardial damage. This may be partly attributable to the fact that in the left anterior oblique the view is from the apex of the ventricle rather than perpendicular to the long axis, leading to a foreshortening effect. This potential limitation in the ability of the left anterior oblique to quantify the extent of akinesis should be borne in mind when the left anterior oblique projection is used alone in gated blood-pool scanning. The correlation coefficients obtained from the single plane right anterior oblique and the biplane are identical $(0.81)$ and are similar to the correlations obtained by Feild et al. (1972) and Rigo et al. (1974). One limitation in this relation of akinetic segment size to depression of overall function is that no consideration is given to the function of the non-akinetic muscle. If the latter is normal the ejection fraction may be maintained at a higher level than if it were hypokinetic. Secondly, since measurements were performed in two projections in two dimensions, the akinetic percentage can only be an estimate of the true akinetic area of the left ventricle. Despite these limitations, the extent of akinesis is a reflection of the size of an infarct. With the evidence that infarct size is an important factor in prognosis (Sobel et al., 1972), radionuclide ventriculography may become a new scintigraphic approach to the problem of assessing infarct size.

In summary, right anterior oblique and left anterior oblique first pass radionuclide ventriculograms performed on patients in the convalescent phase of acute myocardial infarction can provide information on ejection fraction and absolute ventricular volumes, as well as allowing visualisation of all areas of the left ventricle. The detection of abnormally contracting segments is superior for the combined projections than for a single plane study, a factor especially important in patients with multiple areas of infarction electrocardiographically. The ability of this non-invasive technique to relate derangements of ventricular structure to those of ventricular function provides a rational basis for the use of 'biplane' radionuclide ventriculography in the assessment of patients with acute myocardial infarction.

The authors are grateful to Mrs J. Wadsworth for help with the statistical analysis.

\section{References}

Bodenheimer, M. M., Banka, V. S., Fooshee, C. M., Hermann, G. A., and Helfant, R. H. (1978). Quantitative radionuclide angiography in the right anterior oblique view: comparison with contrast ventriculography. American fournal of Cardiology, 41, 718-725.

Budinger, T. F., and Rollo, F. D. (1977). Physics and instrumentation. Progress in Cardiovascular Diseases, 20, $19-53$.

Cohn, P. F., Gorlin, R., Adams, D. F., Chahine, R. A., Vokonas, P. S., and Herman, M. V. (1974). Comparison of biplane and single plane left ventriculograms in patients with coronary artery disease. American fournal of Cardiology, 33, 1-6.

Dymond, D. S., Jarritt, P. H., Britton, K. E., and Spurrell, R. A. J. (1979). Detection of post-infarction left ventricular aneurysms by first pass radionuclide ventriculography using a multicrystal gamma camera. British Heart fournal, 41, 68-78.

Feild, B. J., Russell, R. O., jun, Dowling, J. T., and Rackley, C. E. (1972). Regional left ventricular performance in the year following myocardial infarction. Circulation, 46, 679-689.

Garcia, E., Sardi, E., Hammer, S., Koorji, A., Mallan, S., and Gottlieb, S. (1977). A method for isolating the left ventricle in the right anterior oblique projection from equilibrium gated blood pool radionuclide imaging (abstract). Circulation, 55 and 56, Suppl III, 53.

Greene, D. G., Carlisle, R., Grant, C., and Bunnell, I. L. (1967). Estimation of left ventricular volume by one plane cineangiography. Circulation, 35, 61-69.

Henning, H., Schelbert, H., Crawford, M. H., Karliner, J. S., Ashburn, W., and O'Rourke, R. A. (1975).Left ventricular performance assessed by radionuclide angiocardiography and echocardiography in patients with previous myocardial infarction. Circulation, 52, 1069-1075.

Herman, M. V., Heinle, R. A., Klein, M. D., and Gorlin, R. (1967). Localised disorders in myocardial contraction. Asynergy and its role in congestive heart failure. New England fournal of Medicine, 227, 222232.

Kostuk, W. J., Ehsani, A. A., Karliner, J. S., Ashburn, W. L., Peterson, K. L., Ross, J., and Sobel, B. E. (1973). Left ventricular performance after myocardial infarction assessed by radioisotopic angiocardiography. Circulation, 47, 242-249. 
Marshall, R. C., Berger, H. J., Costin, J. C., Freedman, G. S., Wolberg, J., Cohen, L. S., Gottschalk, A., and Zaret, B. L. (1977). Assessment of cardiac performance with quantitative radionuclide angiocardiography. Sequential left ventricular ejection fraction, normalised left ventricular ejection rate and regional wall motion. Circulation, 56, 820-829.

Mathey, D., Bleifeld, W., Hanrath, P., and Effert, S. (1974). Attempt to quantitate relation between cardiac function and infarct size in acute myocardial infarction. British Heart fournal, 36, 271-279.

Miller, R. R., Amsterdam, E. A., Bogren, H. G., Massumi, R. A., Zellis, R., and Mason, D. T. (1974). Electrocardiographic and cineangiographic correlations in assessment of the location, nature and extent of abnormal left ventricular segmental contraction in coronary artery disease. Circulation, 49, 447-454.

Pairolero, P. C., Hallerman, F. J., and Ellis, F. H., jun (1970). The left ventriculogram in experimental myocardial infarction. Radiology, 95, 311-316.

Pierson, R. N., Alam, S., Kemp, H. G., and Friedman, M. I. (1977). Radiocardiography in clinical cardiology. Seminars in Nuclear Medicine, 7, 85-100.

Rackley, C. (1976). Quantitative evaluation of left ventricular function by radiographic techniques. Circulation, 54, 862-879.

Rigo, P., Murray, M., Strauss, H. W., Taylor, D., Kelly, D., Weisfeldt, M., and Pitt, B. (1974). Left ventricular function in acute myocardial infarction evaluated by gated scintigraphy. Circulation, 50, 678-684.

Schelbert, H. R., Henning, H., Ashburn, W. L., Verba, J. W., Karliner, J. S., and O'Rourke, R. A. (1976). Serial measurements of left ventricular ejection fraction by radionuclide angiography early and late after myocardial infarction. American fournal of Cardiology, 38, 407-415.

Sobel, B. E., Bresnahan, G. F., Shell, W. E., and Yoder, R. D. (1972). Estimation of infarct size in man and its relation to prognosis. Circulation, 46, 640-648.

Steele, P., Kirch, D., Matthews, M., and Davies, H. (1974). Measurement of left heart ejection fraction and end diastolic volume by a computerised scintigraphic technique using a wedged pulmonary arterial catheter. American fournal of Cardiology, 34, 179-186.

Strauss, H. W., Zaret, B. L., Hurley, P. J., Natarajan, T. K., and Pitt, B. (1971). A scintiphotographic method for measuring left ventricular ejection fraction in man without cardiac catheterisation. American fournal of Cardiology, 28, 575-580.

Tennant, R., and Wiggers, C. J. (1935). Effect of coronary occlusion on myocardial contraction. American fournal of Physiology, 112, 351-361.

Van Dyke, D., Anger, H. O., Sullivan, R. W., Vetter, W. R., Yano, Y., and Parker, H. G. (1972). Cardiac evaluation from radioisotope dynamics. fournal of Nuclear Medicine, 13, 585-592.

Wynne, J., Green, L. H., Mann, T., Levin, D., and Grossman, W. (1978). Estimation of left ventricular volumes in man from biplane cineangiograms filmed in oblique projections. American fournal of Cardiology, 41, 726-732.

Requests for reprints to Dr D. S. Dymond, Department of Cardiology, St. Bartholomew's Hospital, London EC1A 7BE. 